\title{
SNP-ChIP: a versatile and tag-free method to quantify changes in protein binding across the genome
}

\author{
Luis A. Vale-Silva ${ }^{1,2}$, Tovah E. Markowitz ${ }^{1}$ and Andreas Hochwagen ${ }^{1 *}$ (D)
}

\begin{abstract}
Background: Chromatin-immunoprecipitation followed by sequencing (ChIP-seq) is the method of choice for mapping genome-wide binding of chromatin-associated factors. However, broadly applicable methods for between-sample comparisons are lacking.

Results: Here, we introduce SNP-ChIP, a method that leverages small-scale intra-species polymorphisms, mainly SNPs, for quantitative spike-in normalization of ChIP-seq results. Sourcing spike-in material from the same species ensures antibody cross-reactivity and physiological coherence, thereby eliminating two central limitations of traditional spike-in approaches. We show that SNP-ChIP is robust to changes in sequencing depth and spike-in proportions, and reliably identifies changes in overall protein levels, irrespective of changes in binding distribution. Application of SNP-ChIP to test cases from budding yeast meiosis allowed discovery of novel regulators of the chromosomal protein Red 1 and quantitative analysis of the DNA-damage associated histone modification $\mathrm{\gamma}-\mathrm{H} 2 \mathrm{AX}$.
\end{abstract}

Conclusion: SNP-ChIP is fully compatible with the intra-species diversity of humans and most model organisms and thus offers a general method for normalizing ChIP-seq results.

Keywords: Chromatin immunoprecipitation, ChIP-seq, Spike-in, Normalization, Chromosomal proteins, Post-translational modification, Meiosis, S. cerevisiae

\section{Background}

Chromatin immunoprecipitation followed by DNA sequencing (ChIP-seq) has emerged as the method of choice for mapping the genome-wide distribution of proteins and protein modifications and has led to important discoveries in both basic chromatin biology and disease states $[1,2]$. A core result of ChIP-seq experiments is the generation of genome-wide target signal tracks, which are obtained from read pileups, typically normalized against a mock, non-immunoprecipitated control sample (input sample). Signal tracks are used for identification of regions with elevated numbers of mapped reads (peaks) as well as other downstream analyses [3]. However, because of the necessary internal normalization procedures, signal tracks can only be used for comparisons between samples if a method for inter-sample normalization is

\footnotetext{
* Correspondence: andi@nyu.edu

'Department of Biology, New York University, New York, NY 10003, USA

Full list of author information is available at the end of the article
}

available [3]. This is a crucial, often overlooked, caveat of ChIP-seq, as well as other genome-wide biochemical analysis methods relying on next-generation sequencing [4].

For sparsely bound proteins, such as transcription factors, inter-sample normalization can often be achieved using statistical methods [2] or ChIP followed by real-time quantitative PCR (ChIP-qPCR) [5]. These methods, however, either assume constant global signal or a constant signal at selected genes as basis for normalization, which is difficult to verify, in particular for more broadly distributed factors. The solution to overcome this limitation is the addition of a "spike-in" reference sample [2, 6]. The spikein procedure consists of adding a constant amount of exogenous material to all tested samples, ideally prior to any critical steps in the experimental protocol. Provided that the spike-in material contains a target that is bound by the antibody as efficiently as the study target and that the resulting sequencing reads can be distinguished from the test sample, the number of spike-in reads should be the same across all tested samples. The spike-in thus functions 
as an internal control against which to normalize the ChIPseq results [6]. Spike-ins are well established for RNA-seq analyses where use of RNA from a different species allows simple sequence-based distinction between test sample and spike-in [7]. The additional requirement for cross-reactivity of the antibody in ChIP-seq experiments, however, effectively restricts the applicability of inter-species spike-ins to a limited set of highly conserved proteins. For example, one previous work targeted subunits of RNA polymerases II and III in mouse chromatin and spiked with human chromatin [8]. To ensure cross-reactivity, both antibodies were raised against peptides that are $100 \%$ conserved between mouse and human. Another study successfully measured global changes in post-translational demethylation of lysine 79 of histone H3 in human cells, using a Drosophila melanogaster cell spike-in [9].

Ways to broaden the applicability of ChIP spike-ins include either tagging proteins in the test and spike-in samples with a common epitope [10], or using a second, spike-in specific antibody against a natural [11] or a synthetic target [12]. These strategies, however, come with their own specific drawbacks. The use of protein tagging adds the potential for prohibitive disruption of protein function and is incompatible with the analysis of protein modifications. The use of a second, spike-in specific antibody, on the other hand, requires labor-intensive technical validation of the compatibility of the second antibody and no longer controls for biases in the immunoprecipitation step between samples.

Here, we show that these issues can largely be overcome by using spike-in material from the same species. This approach, which we name SNP-ChIP, enables reproducible semi-quantitative measurement of global protein levels and also works for protein modifications and fast evolving proteins.

\section{Results}

\section{Experimental rationale of SNP-ChIP}

The basic premise of SNP-ChIP is that cells from the same species can serve as spike-in material provided they harbor sufficient genetic diversity, primarily in the form of single-nucleotide polymorphisms (SNPs). The signal at each polymorphism provides an independent measure of test-sample/spike-in ratio that together allows calculation of a normalization factor and appropriate scaling of ChIP-seq results (Fig. 1a). If there is sufficient genetic diversity to allow a large fraction of sequencing reads to be assigned to the genomes of origin, SNP-ChIP additionally allows the generation of genome-wide target distribution profiles. Importantly, because SNP-ChIP uses the same species as the source of the spike-in material, it will work with virtually any target in the organism's proteome, including post-translational modifications, provided a ChIP-grade antibody is available.

\section{SNP-ChIP of a rapidly evolving chromosomal protein}

To test the utility of intra-species spike-ins, we turned to chromatin analyses in yeast. We specifically focused on chromosomes in meiosis because this process involves many broadly distributed chromosomal proteins and posttranslational modifications. One typical example is the axial-element protein Red1, which plays important roles in meiotic recombination. Red1 is broadly bound along chromosomes [13-15] but, like other meiotic factors, its sequence has diverged even in closely related species [16]. Furthermore, like many proteins, Red1 cannot easily be tagged without disrupting protein function $[15,17]$. These attributes mean Red1 is not amenable to standard spike-in approaches, making it a particularly suitable target for SNP-ChIP. Moreover, mutations that change the overall levels and chromosomal distribution of Red1 are available $[15,18,19]$, providing benchmarks for evaluating the efficacy of SNP-ChIP.

SNP-ChIP of Red1 was performed using the SK1 genetic background [20] as test strain and a meiosis-optimized variant of the widely used S288c reference strain as spike-in [21, 22]. For both genetic backgrounds, high-quality end-to -end genome assemblies are available [23]. These assemblies differ by about 76,000 SNPs, spaced at an overall median distance of $70 \mathrm{bp}$ (Additional file 1: Figure S1a) consistently across all chromosomes (Additional file 1: Figure S1b), which constitutes enough variation to allow unambiguous assignment of a large proportion of sequencing reads. To perform SNP-ChIP, test cells (SK1) were mixed with a constant fraction of meiotic spike-in cells (S288c) before subjecting the mixtures to a standard ChIPseq protocol. The generated reads were aligned to a hybrid genome built by concatenating genome assemblies of the test and spike-in genomes. Reads were aligned with perfect match conditions, excluding any reads aligning to more than one location. Consequently, any reads overlapping at least one SNP were assigned to a specific genome and genomic location, while reads not overlapping a polymorphism mapped to both genomes and were thus discarded.

We initially investigated the ability of SNP-ChIP to detect changes in chromatin association resulting from reduced protein production. The red $1_{y c s} 4 S$ allele is caused by a mutation in the promoter of RED1 that leads to a reduction of Red1 levels to about $20-25 \%$ of wild type and a near complete loss of cytologically observable axial elements [19]. Importantly, traditional ChIP-seq analysis was unable to detect this change in protein abundance and produced indistinguishable Red1 profiles between wild type and red $1_{y c s 4 S}$ mutants [19]. By contrast, when we applied SNP-ChIP to compare these two strains, the reduced Red1 binding levels were readily apparent (Fig. 1b). Calculation of a spike-in normalization factor based on the relative abundance of total sample and spike-in reads yielded a Red1 level in the red $1_{y c s 4 S}$ mutant of $28.8 \pm 5.1 \%$ (S.D.) 

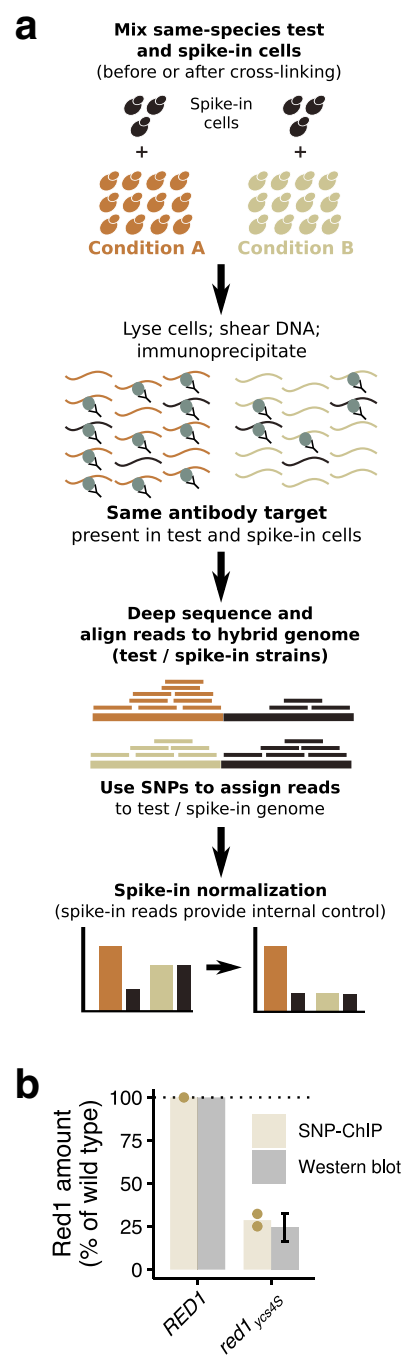
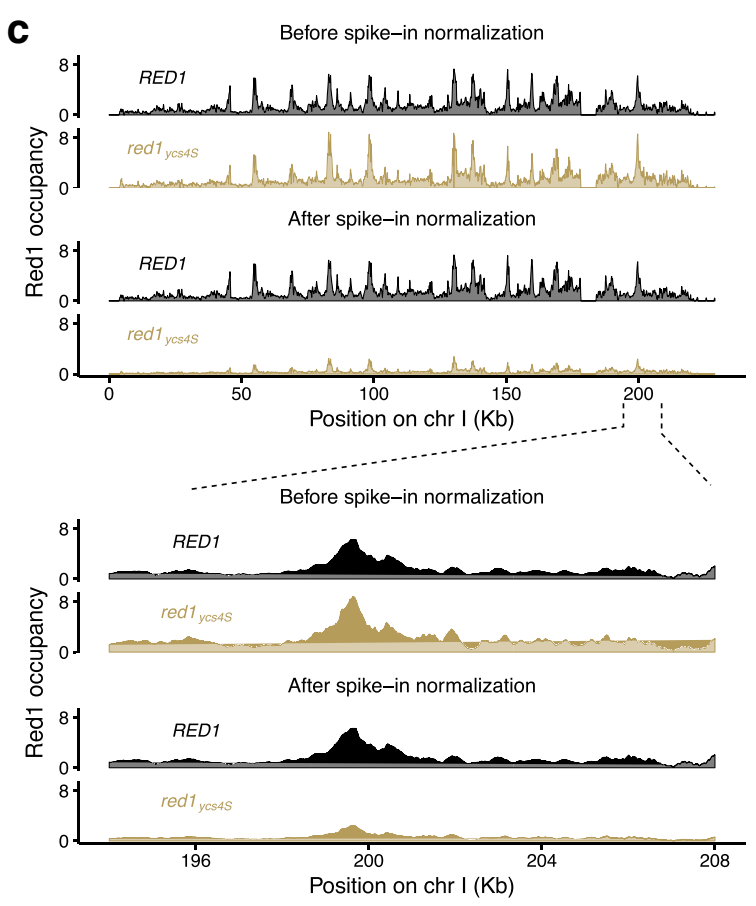

d

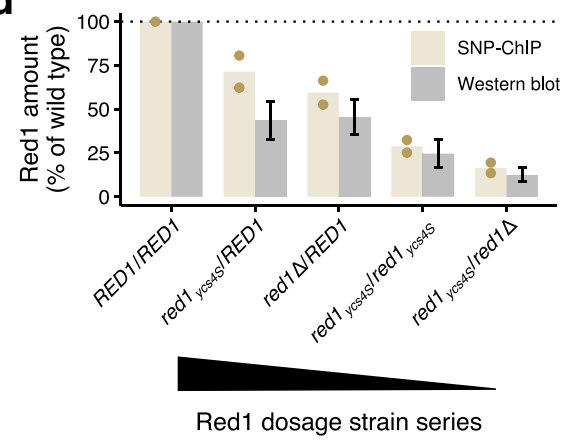

Fig. 1 SNP-ChIP adds the ability to measure semi-quantitative amounts of target protein to traditional ChIP-seq. a Main steps of SNP-ChIP exemplified for two hypothetical conditions. b Target protein Red1 levels produced by SNP-ChIP (equivalent to the wild type-normalized spike-in normalization factor), compared to previously published levels measured by western blot (mean +/- S.E.M.) [19]. Points represent individual SNPChIP-derived replicate values and bars represent average value. c Fragment pileup produced using MACS2 with SPMR (fragment pileup per million reads) sequencing depth normalization of an example chromosome and chromosomal region before (top panel) and after (bottom panel) spike-in normalization. $\mathbf{d}$ Target protein Red1 levels for Red1 dosage strain series compared to previously published levels measured by western blot (mean +/- S.E.M.) [19], as in (b)

of the wild type, closely matching the reported change in Red1 levels obtained from western analysis [19]. This normalization factor allowed appropriate signal scaling of ChIP-seq profiles for the two conditions (Fig. 1c).

SNP-ChIP was further validated by applying it to a Red1 dosage series, which consists of different combinations of RED1 alleles (RED1, red $1_{y c s} 4$, red $\left.1 \Delta\right)$ yielding a stepwise decrease in Red1 levels (Fig. 1d) [19]. SNP-ChIP measurements of Red1 chromatin association in this series again closely matched previously published protein levels (Fig. 1d). In fact, SNP-ChIP measurements appeared more accurate than quantitative western analysis, which failed to resolve the expected reduction in protein levels between $R E D 1 /$ red $1_{y c s} 4 S$ and $R E D 1 /$ red1 $1 \Delta$ cells [19]. Taken together, these data show that SNP-ChIP accurately measures reductions in global Red1 binding over a wide range of target protein levels.

\section{SNP-ChIP is robust to variation in sequencing depth and fraction of spike-in cells}

We used several approaches to probe the technical robustness of SNP-ChIP. High-throughput sequencing technologies produce variable numbers of reads per sample, depending on factors like sequencing instrument and 
sample mutiplexing. To model the effect of lower sequencing depth on the reproducibility of SNP-ChIP analysis, we subsampled the reads of the immunoprecipitated and input samples from wild type and red $1_{y c s} 4 S$ test conditions to different depths (ranging from 1 to 10 million reads). Plotting subsample size against number of aligned reads showed a perfectly linear correlation for all samples (Fig. 2a), indicating that a wide range of sequencing depths will yield robust quantitative information by SNP-ChIP. For these particular test conditions, the fraction of reads mapping to a specific genome, and thus kept in the analysis, was around $20 \%$ for the wild type and $30 \%$ for the $\operatorname{red} 1_{\text {ycs } 4 S}$. We computed the spike-in normalization factor using all 10,000 possible combinations of read subsamples (10 read subsamples ranging from 1 to 10 million reads for each of four sequenced samples: wild type ChIP and input samples plus red $1_{y c s 4 S}$ ChIP and input samples) and found a very tight distribution of results $(0.2848 \pm 0.0015$, S.D.; Fig. 2b). This establishes that sequencing depth does not need to be balanced between immunoprecipitated and input samples, or between different conditions, to produce accurate proportions of reads mapping to the test and the spike-in genomes.

Another condition that may affect the results of SNPChIP is the amount of spike-in material added to the samples. Spike-in normalization methods assume a linear relationship between the amount of spike-in material and the resulting proportion of spike-in reads in the immunoprecipitated sample. This condition is essential for the results to be independent of the amount of spike-in material. To verify this assumption, we prepared samples with spike-in cell proportions ranging from 5 to $30 \%$. As test samples we used wild type and a strain with a single red1-pG162A promoter mutation that phenocopies the red1 $1_{y c s}$ S allele. While red $1_{y c s 4 S}$ contains an introgressed genomic region with dozens of SNPs surrounding the RED1 locus, the red1-p $162 A$ mutant was engineered to carry only the specific mutation responsible for the reduction in Red1 levels [19]. As shown in Fig. 2c, the proportion of spike-in reads in the input samples (reflecting the amount of spike-in
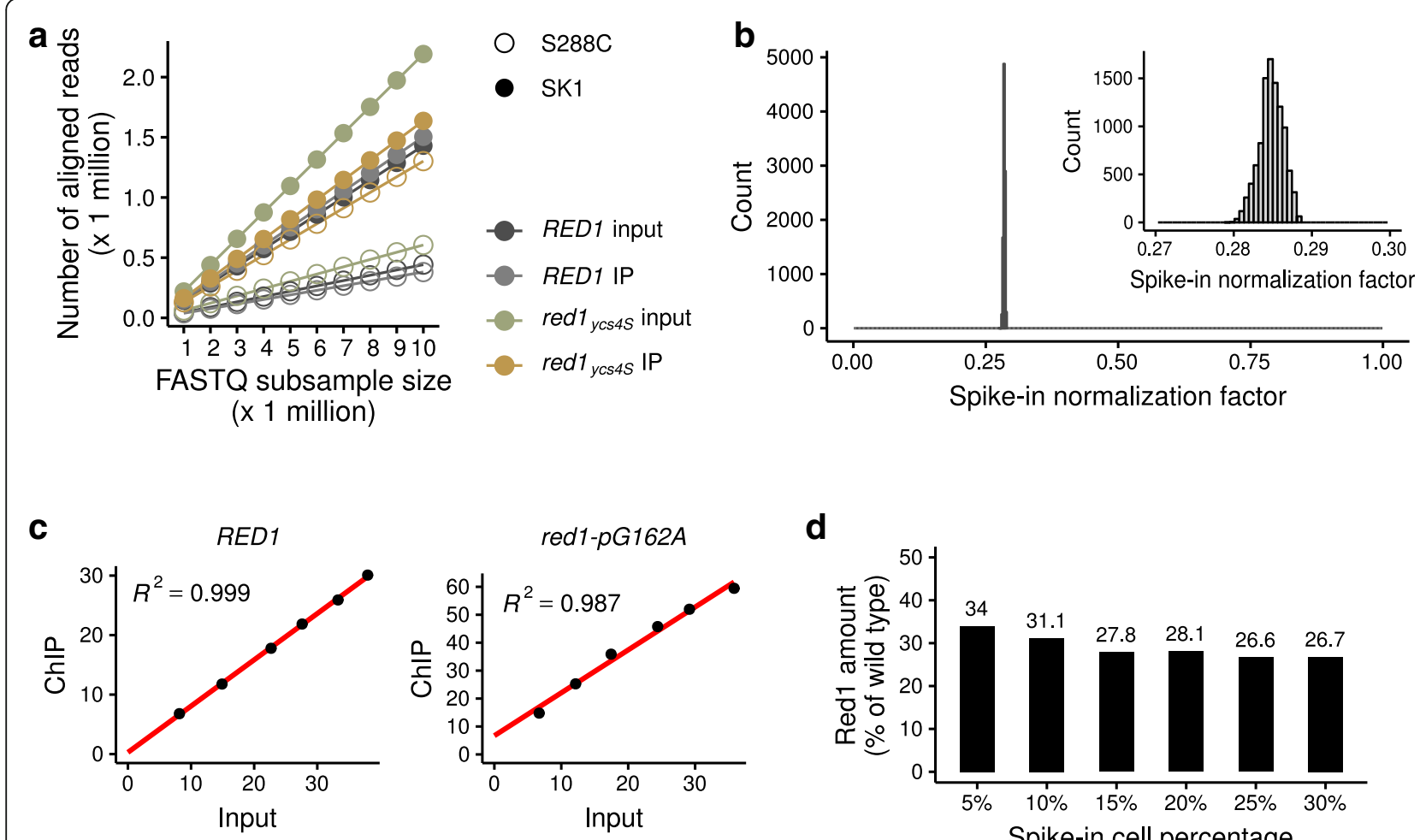

c

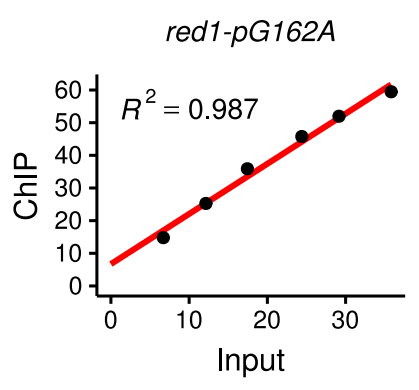

d

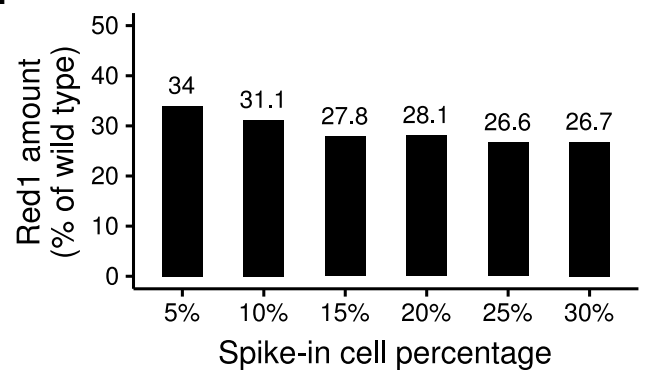

Fig. 2 SNP-ChIP is robust to variation in both sequencing depth and amount of spike-in cells. a Number of aligned reads in SNP-ChIP as a function of total size of raw read sample. Systematic raw read subsamples of size 1 to 10 million were obtained for each sample and mapped to the hybrid SK1-S288c genome. b Distribution of spike-in normalization factors computed using all 10,000 possible combinations of read subsamples in (a). The inset is a zoom-in on a narrow window of the $x$ axis where all values are located. $\mathbf{c}$ Percentage of reads aligning to the spike-in genome in the input sample versus the immunoprecipitated sample (ChIP) for wild type and red1-pG162A strains. Note: In contrast to red $1_{y c s 45}$, which contains an introgressed region with dozens of SNP surrounding the RED1 locus, the red1-pG162A mutant only carries the causative promoter mutation. d Resulting wild type-normalized spike-in normalization factor (equivalent to Red1 amount) in the red1-pG162A strain after performing SNP-ChIP with percentages of added spike-in cells ranging from 5 to $30 \%$. The results suggest that spike-in material proportions of $15 \%$ and higher are appropriate for SNP-ChIP 
material added to the test sample) correlates linearly with the resulting proportion of spike-in reads in the immunoprecipitated sample, for both the wild type and the red1-$p G 162 A$ sample. Moreover, the red1-pG162A sample yielded a very similar Red 1 amount to the red $1_{y c s 4 S}$ allele ( $28.8 \%$ versus $28.1 \%$ of wild type, respectively, when using $20 \%$ of spike-in cells), further supporting the robustness of the method. Low spike-in cell percentages (5 and 10\%) resulted in somewhat increased estimates of the Red1 amount (Fig. 2d), likely due to increased noise. These results suggest that spike-in material proportions of $15 \%$ and higher are appropriate for SNP-ChIP. All other experiments shown here used a spike-in proportion of $20 \%$.

Finally, we investigated the impact of the calculation method to compute the spike-in normalization factor. The SNP-ChIP normalization factor calculated in the examples shown so far relies on total read counts aligned to the test and the spike-in genomes. An alternative method is to compute the scalar mean value of the aligned read pileup score. We tested the utility of this alternative by calculating the pileup score at (1) all genomic positions, (2) at SNP positions only, or (3) at SNP positions falling within called signal peaks (see Methods section). The last approach will effectively exclude regions expected to hold only background signal, along with any false negative regions. We found very similar values and high concordance between all four methods in all cases (Additional file 2: Figure S2a), although read pileups consistently produce slightly lower values than the read count method (Additional file 2: Figure S2b). Overall, however, the difference is relatively small and we believe the read count-based method, which is computationally much simpler, represents an appropriate approximation, at least for broadly distributed proteins.

\section{Binding profiles obtained directly from SNP-ChIP experiments}

The primary utility of SNP-ChIP is the generation of a normalization factor that allows scaling of profiles obtained by traditional ChIP-seq experiments run under the same conditions (Fig. 1c). Given the broad distribution of SNPs across the two analyzed genomes, we explored the possibility that SNP-ChIP could also directly yield informative binding profiles, even though this application is clearly limited by the available SNP density. Comparing a sample sequenced with spike-in to data obtained using a replicate, non-spiked sample [15] shows that signal tracks of spiked samples closely mirror those of the non-spiked control, although some signal gaps can be seen in the spiked sample (Additional file 3: Figure S3a; examples indicated by the red arrows). Thus, as expected, the use of same-species spike-in causes some loss of information. This issue appears negligible for broad peaks, as called peaks show a very close agreement (Additional file 3: Figure S3b). Narrow peaks show more disagreement, with only about one third of the called peaks overlapping between the two samples. These data indicate that SNP-ChIP can also provide direct information about protein distribution, in particular for larger-scale binding patterns.

\section{Global Red1 levels are reduced in cohesin and hop 1D mutants}

We sought to apply SNP-ChIP to investigate mutant situations that cause a broad protein redistribution. Redistribution is a challenge for traditional quantification methods, such as ChIP-qPCR, because identifying regions that remain unbound is non-trivial. In the absence of conserved cohesin subunit Rec8, Red1 distribution along the genome changes dramatically, displaying large regions of depletion alternating with dense clusters of binding $[15,18]$. Whether overall binding levels of Red1 change in rec8 $\Delta$ mutants, however, remains unclear. We employed SNP-ChIP to address this question and found a pronounced decrease of overall Red1 binding levels (Fig. 3a). Direct comparison of Red1 occupancy along two example chromosomes illustrates both the dramatic redistribution and the overall decrease in Red1 binding compared to wild type (Fig. 3b). Thus, Rec8-cohesin is essential for the full chromosomal enrichment of Red1.

Hop1 is another important protein of the yeast axial element [24] that physically interacts with Red1 [17]. Axialelement proteins are recruited in higher amounts to small chromosomes, but in the absence of Hop1, Red1 binding becomes less dependent on chromosome size [15]. Previous work using in silico scaling [25], suggested that this reduction resulted from a selective increase in Red1 recruitment to large chromosomes [15]. That scaling approach, however, requires the definition of genomic regions that are unbound, which is difficult to ascertain with broadly distributed chromosomal proteins like Red1. Therefore, we reinvestigated this question by performing SNP-ChIP of Red1 in a hop1D mutant. SNP-ChIP reproduced the previously found weakening of chromosome-size bias. However, the spike-in normalization factor showed an overall decrease of Red1 recruitment to $71.9 \pm 4.2 \%$ of the wild type Red1 amount (Fig. 3c, d). This decrease is stronger on small chromosomes (Fig. 3e). We note that mild loss of Red1 binding does not generally result in a loss of chromosome-size bias, because deletion of the histone methyltransferases Set1 and Dot1 causes similar 20\% reductions of overall Red1 recruitment levels but does not affect the distribution of Red1 binding among chromosomes (Additional file 4: Figure S4). These data suggest that loss of Hop1 leads to a general reduction of Red1 signal across all chromosomes that particularly affects the three smallest chromosomes. 


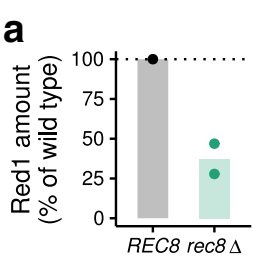

b
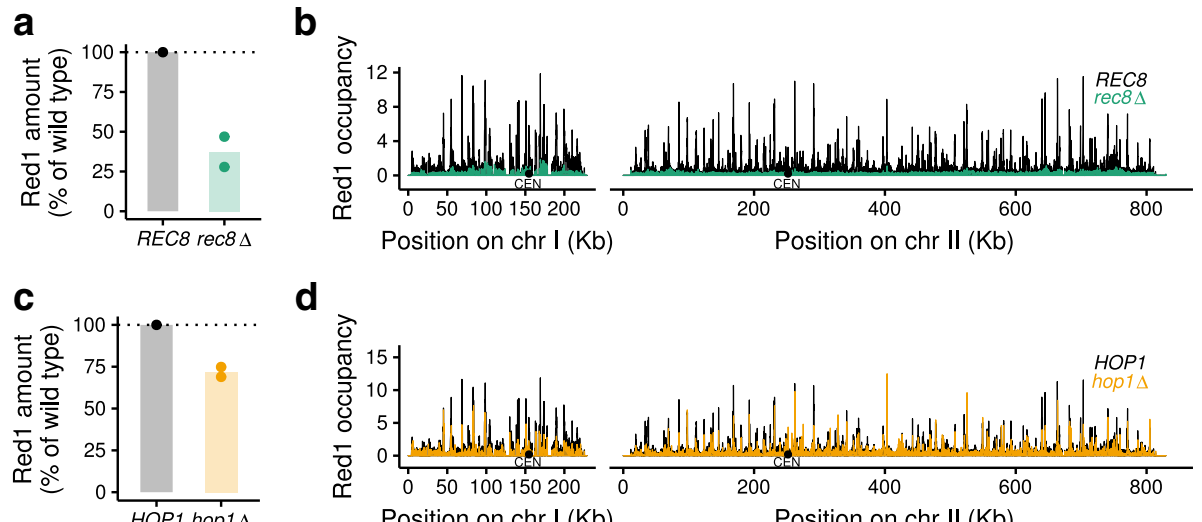

d
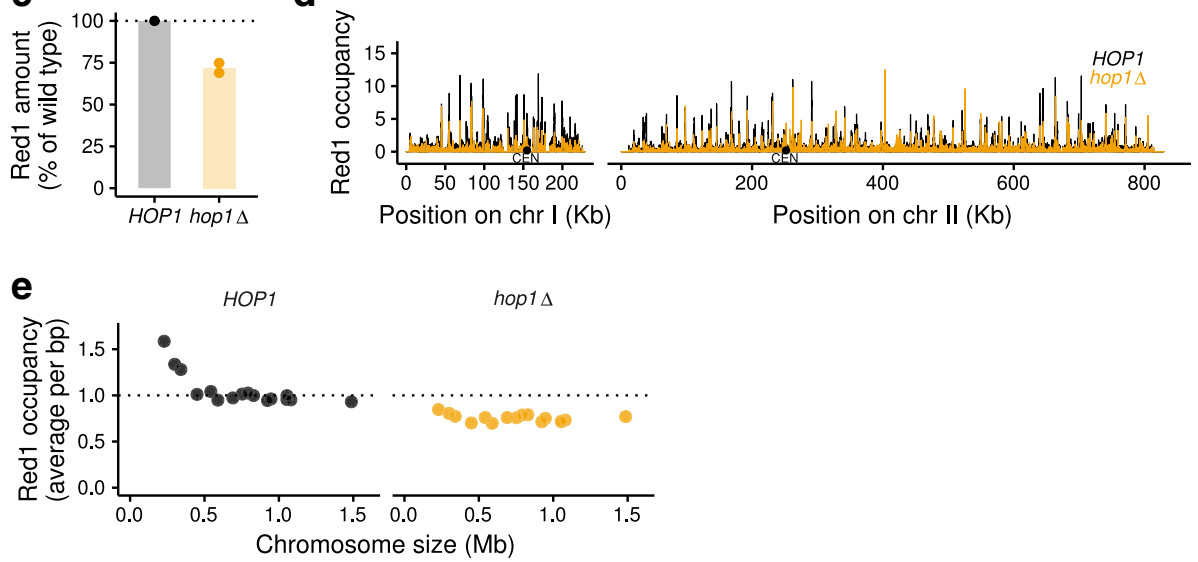

Fig. 3 SNP-ChIP analysis in mutants with large-scale target redistribution. a Target protein Red1 levels in a rec8 $\Delta$ mutant relative to wild type produced by SNP-ChIP. Points represent individual replicate values and bars represent average value. $\mathbf{b}$ Spike-in-normalized fragment pileups in wild type and rec $8 \triangle$ mutant strains produced using MACS2 with SPMR (fragment pileup per million reads) sequencing depth normalization plotted on two example chromosomes. c Red1 levels in hop $1 \Delta$ mutant relative to wild type produced by SNP-ChIP (as in a). d Spike-in-normalized fragment pileups in wild type and hop $1 \Delta$ mutant strains (as in b). e Spike-in-normalized average Red1 signal on individual chromosomes in a hop $1 \Delta$ mutant

$\mathrm{Y}-\mathrm{H} 2 \mathrm{AX}$ levels do not change in Red1 dosage strain series To test if SNP-ChIP also allows quantitative analyses of protein modifications, we targeted phosphorylation of histone $\mathrm{H} 2 \mathrm{~A}$ on serine $129(\gamma-\mathrm{H} 2 \mathrm{AX})$. This modification is rapidly induced following the formation of DNA doublestrand breaks (DSBs) [26]. In mitotic yeast, the $\gamma$-H2AX modification spreads about $50 \mathrm{~kb}$ on either side of a DSB $[27,28]$. In addition, constitutive $\gamma-\mathrm{H} 2 \mathrm{AX}$ is found near telomeres throughout the cell cycle [29]. To analyze the distribution and DSB dependence of $\gamma-\mathrm{H} 2 \mathrm{AX}$ in meiosis, we performed SNP-ChIP in a wild type strain, as well as the Red1 dosage series, which shows a mild (up to 30\%) reduction in DSB levels [19], and a spo11-Y135F mutant, encoding a catalytically dead Spo11 protein, which does not form meiotic DSBs [30, 31].

Measuring $\gamma-\mathrm{H} 2 \mathrm{AX}$ levels in meiosis revealed no difference between wild type and any of the strains with reduced Red1 levels, irrespective of calculation method (Fig. 4a, b, c). The uniform signal along chromosomes is consistent with the spreading of the $\gamma$-H2AX mark from all yeast DSB hotspots, which are distributed throughout the whole genome, and likely explains why a mild reduction in DSB levels does not lead to a noticeable drop in global $\gamma$-H2AX signal. The spo11-Y135F control, on the other hand, displayed only about $25 \%$ of the wild type $\gamma$-H2AX levels. Signal was markedly enriched next to telomeres, with the interstitial regions only showing weak signals likely associated with gene expression [32]. These data show that the constitutive telomere-associated $\gamma$-H2AX signal is also maintained in meiotic prophase. Moreover, scaling of signal tracks indicates that telomere-adjacent $\gamma$-H2AX signal remains largely unchanged in the spo11-Y135F mutant, consistent with the fact that meiotic DSB formation is nearly undetectable in these regions [33]. Together, these data show that SNP-ChIP allows quantitative comparisons between ChIP-seq experiments regardless of the antigen and thus provides a versatile method for measuring global chromatin associations without the need for epitope tags.

\section{Discussion}

Our data show that small-scale intra-species genetic polymorphisms can be leveraged for quantitative spike-in normalization of ChIP-seq results. Sourcing spike-in material from the same species largely preserves antibody cross-reactivity and thus will work with virtually any target in an organism's proteome without the need for epitope tagging. It also ensures complete physiological coherence between the test and the spike-in cells, thereby avoiding biases at experimental steps such as chromatin fixation or cell lysis. 


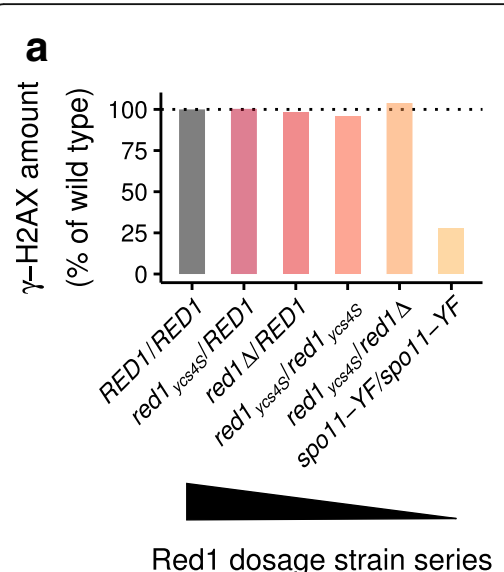

\section{b}

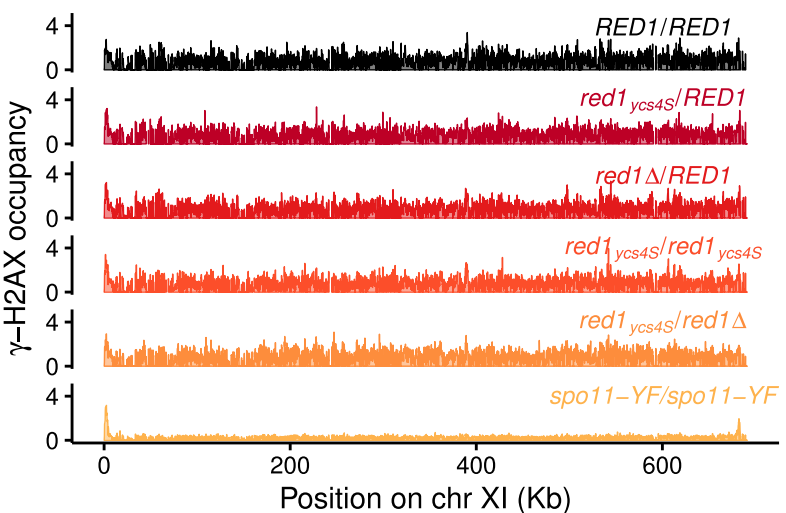

C

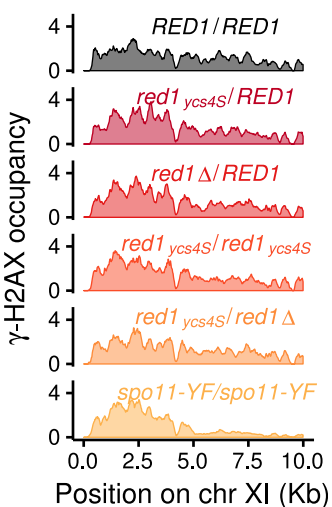

Fig. 4 SNP-ChIP analysis of a protein modification. a $y-H 2 A X$ levels for the Red1 dosage strain series and a spo11-Y135F mutant relative to wild type produced by SNP-ChIP. b Fragment pileup produced using MACS2 with SPMR (fragment pileup per million reads) sequencing depth normalization on an example chromosome after spike-in normalization. c Detail of data in (b) showing a zoom-in on the left subtelomeric region

The primary output of SNP-ChIP is a normalization factor that can be used to appropriately scale ChIP-seq profiles. Because the normalization factor relies on combined measurements of thousands of SNPs it is highly robust to variations in sequencing depth or changes in protein distribution between samples. In multiplexed libraries, SNP-ChIP can therefore be performed with relatively low sequencing coverage alongside traditional ChIP-seq experiments to yield the necessary scaling information.

SNP-ChIP can also provide substantial positional information, although this application is necessarily limited by the availability of high-confidence SNPs. Our experiments using yeast strains with $\sim 0.7 \%$ sequence divergence and 100-nt long reads showed that the method generated sufficient resolution to recover genomic regions of Red1 enrichment. Moreover, preliminary experiments indicate that using longer reads further minimizes gaps (data not shown). Thus, SNP-ChIP can provide high-quality pilot information for subsequent ChIP-seq analyses at higher read depth.

The reliance on thousands of SNPs also means that SNP-ChIP will be particularly powerful for the quantitative analysis of broadly distributed proteins and chromatin marks. Applying SNP-ChIP to proteins that interact with chromatin in more specific, highly localized positions (e.g. transcription factors), will likely result in a disproportionate number of SNPs exhibiting background signal that will affect the calculation of the normalization factor. Indeed, preliminary experiments testing the budding yeast transcription factor Gal4 suggested that SNP-ChIP is not ready to handle such targets. While SNP-ChIP generated reliable signal track data, the normalization factor computation method does not work as-is and failed to detect differences in overall Gal4 binding (data not shown). SNP-ChIP would thus require further development to be usable with sparsely binding proteins. We note, however, that these are inherently more tractable targets for ChIP-qPCR, thus reducing the need for a spike-in method.

\section{Conclusion}

We conclude that SNP-ChIP provides a versatile method for normalizing the ChIP-seq results of broadly distributed chromosomal proteins and post-translational modifications. SNP-ChIP is fully compatible with the intra-species genetic diversity of humans and most model organisms [34] and should be applicable to any experimental system for which a reliable collection of high-quality SNPs is available. In preliminary in silico experiments testing decreasing numbers of SNPs, the method generated stable normalization factors with as low as $0.01 \%$ sequence divergence (equivalent to about 1200 SNPs in the yeast genome; data not shown). Thus, we expect that SNP-ChIP will allow semiquantitative mapping of a wide range of chromatin binding factors and modifications that have so far stood beyond the reach of quantitative ChIP-seq methods.

\section{Best practice for SNP-ChIP}

The chief prerequisite for successful SNP-ChIP normalization is the availability of high-quality genome assemblies for two different strains or cell lines of the same species, as well as a ChIP-grade antibody against the ChIP target. For optimal results, we recommend using a minimum of $15 \%$ of spike-in material and at least 100-bp sequencing read length. Using longer reads will increase the proportion of assigned reads and minimize signal gaps. In general, SNP-ChIP should be used alongside traditional ChIP-seq experiments. This setup retains the maximal spatial resolution provided by ChIP-seq while providing the necessary scaling factor for quantitative 
comparisons between samples. Relatively shallow sequencing coverage of the SNP-ChIP sample is sufficient for this purpose. In addition, SNP-ChIP also serves as a stand-alone method for exploratory purposes that do not require $<100 \mathrm{bp}$ resolution.

\section{Methods}

\section{Strains and meiotic time courses}

All strains used are listed in Additional file 5: Table S1. The test-sample strains were of the SK1 background. The spike-in material used a meiosis-optimized S288c strain that carries three SK1-derived SNPs, which improve sporulation efficiency and meiotic synchrony of S288c [22]. To further improve synchrony of the spike-in strain, auxotrophic markers were restored using plasmid insertions or PCR-based allele transfer. To induce meiosis, cells were pregrown in YPD for $24 \mathrm{~h}$ at room temperature, followed by inoculation in BYTA media at O.D. $600=0.3$ and growth for $16.5 \mathrm{~h}$ at $30^{\circ} \mathrm{C}$ [35]. Cells were washed twice with water and inoculated at O.D. ${ }_{600}=1.9$ in $0.3 \%$ potassium acetate ( $\mathrm{pH} 7.0)$ to induce meiotic entry. Synchronous entry was confirmed by taking hourly samples for flow cytometry analysis of DNA content.

\section{SNP-ChIP sample preparation}

Samples were collected at $3 \mathrm{~h}$ for SK1 strains or at $6 \mathrm{~h}$ for the slower sporulating S288c spike-in sample. Cells were fixed in $1 \%$ formaldehyde for $30 \mathrm{~min}$ at room temperature and quenched by addition of glycine to a final concentration of $125 \mathrm{mM}$. For the experiments shown here, we fixed the spike-in cells in advance as a batch and kept frozen aliquots at $-80^{\circ} \mathrm{C}$. However, spike-in cells can also be prepared simultaneously with the sample cells. The number of cells in each sample was determined by counting on a hemocytometer. Unless indicated otherwise, cells from the test sample (SK1) were mixed with cells from the spike-in sample (S288c) at a ratio of $80 \%: 20 \%$ before cell lysis and ChIP.

\section{Chromatin immunoprecipitation (ChIP) and Illumina sequencing}

ChIP was performed as described previously [12]. Samples were immunoprecipitated with $2 \mu \mathrm{l}$ anti-Red1 serum (Lot\#16440, kind gift of N. Hollingsworth) or $2 \mu \mathrm{l}$ antiphospho-H2A-S129 antibody (Abcam \#ab15083) per sample. Library preparation was performed as described [15]. Library quality was confirmed by Qubit HS assay and 2200 Tape Station. 100-bp single-end sequencing was performed on an Illumina NextSeq 500 instrument.

\section{Read alignment}

The generated reads were aligned to a hybrid genome built by concatenating recently published high-quality genome assemblies of the test and spike-in reference genomes (SK1 and S288c) [23]. Reads were aligned with perfect match conditions and excluding any reads aligning to more than one location. Normalization of read density was completed as described [12]. Where indicated, peaks of enrichment were called using MACS-2.1.0. Plots show an average of two replicates. To evaluate coverage relative to standard ChIP-seq profiles, we compared SNPChIP results to published datasets GSE69232 [15] and GSE87060 [19].

\section{Calculation of the spike-in normalization factor}

SNP-ChIP is based on counts of all reads aligned to each of the endogenous and the spike-in genomes in each sample, computed from the alignment map files in BAM format using SAMtools idxstats (version 1.3.1). Alternatively, the method can use pileup scores, corresponding to read coverage at each genomic position computed from the alignment map files using BEDTools genomecov (version 2.25.0). In this approach, the total count of reads aligned to a sample is replaced by the mean of the genome-wide pileup scores. Three different versions of the mean pileup score were used: computing the mean of (1) the raw pileup scores; (2) the pileup scores at SNP positions only; or (3) the pileup scores at SNP positions falling within identified protein binding regions (signal peaks called using MACS-2.1.0) only. Irrespective of the approach, we define $C_{\text {endo }}$ and $C_{\text {spike }}$ as the calculated values for the endogenous and the spike-in genomes, respectively, in a given sequenced sample. Quotient $Q$ is defined as the ratio between the values:

$$
Q=C_{\text {spike }} / C_{\text {endo }}
$$

The non-immunoprecipitated, input sample's $Q$ value $\left(Q_{\text {Input }}\right)$ provides a measure of the percentage of the sample that is comprised of spike-in cells in the respective test condition. This consists of the experimental percentage of spike-in material actually added to the sample, corrected for any technical variation or imprecision. The ChIP sample's $Q$ value $\left(Q_{C h I P}\right)$ depends on the amount of target protein present in that experimental condition. A normalization factor $N f$ can thus be obtained for each condition as the ratio between the two $Q$ values:

$$
N f=Q_{\text {Input }} / Q_{\text {ChIP }}
$$

To obtain spike-in-normalized conditions, each condition is multiplied by the respective normalization factor value $N f$. The extent to which $Q_{C h I P}$ differs from $Q_{\text {Input }}$ in each experimental condition is determined by the amount of target protein and how much that differs from the amount of target protein in the spike-in. Since the latter is constant across all tested conditions, the 
result of the normalization is a semi-quantitative measure of the target protein amounts, yielding normalized conditions that can be compared directly to each other.

\section{Additional files}

Additional file 1: Figure S1. Distribution of distances between consecutive single-nucleotide polymorphisms (SNPs) found between the SK1 and S288c yeast genomes. (PDF $47 \mathrm{~kb}$ )

Additional file 2: Figure S2. Spike-in normalization factors calculated using different input data types. (PDF $136 \mathrm{~kb}$ )

Additional file 3: Figure S3. Same-species spike-in causes loss of information but does not affect the overall target distribution patterns. (PDF $440 \mathrm{~kb}$ )

Additional file 4: Figure S4. Red1 occupancy is mildly decreased in histone methyltransferase mutants relative to wild type. (PDF $280 \mathrm{~kb}$ )

Additional file 5: Supplementary Material Contains legends for Figures S1 - S4 and Table S1 (Strains used in this study).. (DOCX $30.0 \mathrm{~kb}$ )

\section{Abbreviations}

bp: base pairs; BYTA: Buffered yeast extract/tryptone/acetate; ChIP: Chromatin immuno-precipitation; ChIP-qPCR: ChIP followed by quantitative PCR; ChIPseq: ChIP followed by next-generation DNA sequencing; DNA: Deoxyribonucleic acids; DSB: DNA double-strand break; kb: kilo base pairs; O.D.600: Optical density at 600 nm; PCR: Polymerase chain reaction; RNA: Ribonucleic acids; RNA-seq: Nextgeneration DNA sequencing of RNA; S.D.: Standard deviation; S.E.M: Standard error of the mean; SNP: Single-nucleotide polymorphism; SPMR: Sequence (fragment) pileup per million reads; YPD: Yeast extract/peptone/dextrose; $Y$-H2AX: Histone 2A phosphorylated on serine 129

\section{Acknowledgements}

We thank F. Winston for sharing strains and N. Hollingsworth for sharing antibodies. We are grateful to S. Ercan and S. Keeney for helpful discussions and the NYU Department of Biology Sequencing Core for technical assistance and debarcoding.

\section{Funding}

This work was supported in part by NIH grants R01 GM111715 and R01 GM123035 and research grant FY16-208 from the March of Dimes Foundation to A.H. The funders had no role in the design of the study, the collection, analysis and interpretation of data, or the writing of the manuscript.

\section{Availability of data and materials}

Data sets have been deposited in NCBI's Gene Expression Omnibus and are accessible through GEO Series accession number GSE115092. Code used for data analysis and producing figures is available on Github (https://github.com/ hochwagenlab/SNP-ChIP).

\section{Authors' contributions}

L.A.V.-S. and A.H. conceived of the study. T.E.M produced key resources. L.A.V.S. conducted the experiments. L.A.V.-S. and A.H. analyzed the data and wrote the manuscript with input from T.E.M. All authors read and approved the final manuscript.

\section{Ethics approval and consent to participate} Not applicable.

\section{Consent for publication}

$$
\text { Not applicable. }
$$

\section{Competing interests}

The authors declare that they have no competing interests.

\section{Publisher's Note}

Springer Nature remains neutral with regard to jurisdictional claims in published maps and institutional affiliations.

\section{Author details}

'Department of Biology, New York University, New York, NY 10003, USA. ${ }^{2}$ Present address: BioQuant Center, Heidelberg University, Heidelberg, Germany.

Received: 2 October 2018 Accepted: 13 December 2018

Published online: 17 January 2019

\section{References}

1. Furey TS. ChIP-seq and beyond: new and improved methodologies to detect and characterize protein-DNA interactions. Nat Rev Genet. 2012; 13(12):840-52.

2. Nakato R, Shirahige K. Recent advances in ChIP-seq analysis: from quality management to whole-genome annotation. Brief Bioinform. 2017;18(2):279-90.

3. Bailey T, Krajewski P, Ladunga I, Lefebvre C, Li Q, Liu T, et al. Practical guidelines for the comprehensive analysis of ChIP-seq data. PLoS Comput Biol. 2013;9(11):e1003326.

4. Chen K, Hu Z, Xia Z, Zhao D, Li W, Tyler JK. The overlooked fact: fundamental need for spike-in control for virtually all genome-wide analyses. Mol Cell Biol. 2015;36(5):662-7.

5. Furusawa Y, Endo TA, Obata Y, Ohara O, Ohno H, Hase K. Pitfalls in global normalization of ChIP-seq data in CD4(+) T cells treated with butyrate: a possible solution strategy. Genom Data. 2014;2:176-80.

6. Meyer CA, Liu XS. Identifying and mitigating bias in next-generation sequencing methods for chromatin biology. Nat Rev Genet. 2014;15(11):709-21.

7. Munro SA, Lund SP, Pine PS, Binder H, Clevert DA, Conesa A, et al. Assessing technical performance in differential gene expression experiments with external spike-in RNA control ratio mixtures. Nat Commun. 2014;5:5125.

8. Bonhoure N, Bounova G, Bernasconi D, Praz V, Lammers F, Canella D, et al. Quantifying ChIP-seq data: a spiking method providing an internal reference for sample-to-sample normalization. Genome Res. 2014;24(7): 1157-68.

9. Orlando DA, Chen MW, Brown VE, Solanki S, Choi YJ, Olson ER, et al. Quantitative ChIP-Seq normalization reveals global modulation of the epigenome. Cell Rep. 2014;9(3):1163-70.

10. Hu B, Petela N, Kurze A, Chan KL, Chapard C, Nasmyth K. Biological chromodynamics: a general method for measuring protein occupancy across the genome by calibrating ChIP-seq. Nucleic Acids Res. 2015;43(20):e132.

11. Egan B, Yuan CC, Craske ML, Labhart P, Guler GD, Arnott D, et al. An alternative approach to ChIP-Seq normalization enables detection of genome-wide changes in histone H3 lysine 27 Trimethylation upon EZH2 inhibition. PLoS One. 2016;11(11):e0166438.

12. Blitzblau HG, Chan CS, Hochwagen A, Bell SP. Separation of DNA replication from the assembly of break-competent meiotic chromosomes. PLoS Genet. 2012;8(5):e1002643.

13. Smith AV, Roeder GS. The yeast Red1 protein localizes to the cores of meiotic chromosomes. J Cell Biol. 1997;136(5):957-67.

14. Blat Y, Protacio RU, Hunter N, Kleckner N. Physical and functional interactions among basic chromosome organizational features govern early steps of meiotic chiasma formation. Cell. 2002;111(6):791-802.

15. Sun X, Huang L, Markowitz TE, Blitzblau HG, Chen D, Klein F, et al Transcription dynamically patterns the meiotic chromosome-axis interface. elife. 2015;4:e07424.

16. Cole F, Keeney S, Jasin M. Evolutionary conservation of meiotic DSB proteins: more than just Spo11. Genes Dev. 2010;24(12):1201-7.

17. de los Santos T, Hollingsworth NM. Red1p, a MEK1-dependent phosphoprotein that physically interacts with Hop $1 \mathrm{p}$ during meiosis in yeast. J Biol Chem. 1999;274(3):1783-90.

18. Panizza S, Mendoza MA, Berlinger M, Huang L, Nicolas A, Shirahige K, et al. Spo11-accessory proteins link double-strand break sites to the chromosome axis in early meiotic recombination. Cell. 2011;146(3):372-83.

19. Markowitz TE, Suarez D, Blitzblau HG, Patel NJ, Markhard AL, MacQueen AJ, et al. Reduced dosage of the chromosome axis factor Red1 selectively disrupts the meiotic recombination checkpoint in Saccharomyces cerevisiae. PLoS Genet. 2017;13(7):e1006928.

20. Kane SM, Roth R. Carbohydrate metabolism during ascospore development in yeast. J Bacteriol. 1974;118(1):8-14.

21. Mortimer RK, Johnston JR. Genealogy of principal strains of the yeast genetic stock center. Genetics. 1986;113(1):35-43.

22. Deutschbauer AM, Davis RW. Quantitative trait loci mapped to singlenucleotide resolution in yeast. Nat Genet. 2005;37(12):1333-40. 
23. Yue JX, Li J, Aigrain L, Hallin J, Persson K, Oliver K, et al. Contrasting evolutionary genome dynamics between domesticated and wild yeasts. Nat Genet. 2017 Jun;49(6):913-24.

24. Hollingsworth NM, Byers B. HOP1: a yeast meiotic pairing gene. Genetics. 1989;121(3):445-62.

25. Liang K, Keles S. Normalization of ChIP-seq data with control. BMC Bioinformatics. 2012;13:199.

26. Kinner A, Wu W, Staudt C, lliakis G. Gamma-H2AX in recognition and signaling of DNA double-strand breaks in the context of chromatin. Nucleic Acids Res. 2008;36(17):5678-94.

27. Shroff R, Arbel-Eden A, Pilch D, Ira G, Bonner WM, Petrini JH, et al. Distribution and dynamics of chromatin modification induced by a defined DNA double-strand break. Curr Biol. 2004;14(19):1703-11.

28. Unal E, Arbel-Eden A, Sattler U, Shroff R, Lichten M, Haber JE, et al. DNA damage response pathway uses histone modification to assemble a double-strand break-specific cohesin domain. Mol Cell. 2004;16(6):991-1002.

29. Kim JA, Kruhlak M, Dotiwala F, Nussenzweig A, Haber JE. Heterochromatin is refractory to gamma-H2AX modification in yeast and mammals. J Cell Biol. 2007;178(2):209-18.

30. Bergerat A, de Massy B, Gadelle D, Varoutas PC, Nicolas A, Forterre P. An atypical topoisomerase II from archaea with implications for meiotic recombination. Nature. 1997:386(6623):414-7.

31. Cha RS, Weiner BM, Keeney S, Dekker J, Kleckner N. Progression of meiotic DNA replication is modulated by interchromosomal interaction proteins, negatively by Spo11p and positively by Rec8p. Genes Dev. 2000;14(4):493-503.

32. Szilard RK, Jacques PE, Laramee L, Cheng B, Galicia S, Bataille AR, et al. Systematic identification of fragile sites via genome-wide location analysis of gamma-H2AX. Nat Struct Mol Biol. 2010;17(3):299-305.

33. Pan J, Sasaki M, Kniewel R, Murakami H, Blitzblau HG, Tischfield SE, et al. A hierarchical combination of factors shapes the genome-wide topography of yeast meiotic recombination initiation. Cell. 2011;144(5):719-31.

34. Leffler EM, Bullaughey K, Matute DR, Meyer WK, Segurel L, Venkat A, et al. Revisiting an old riddle: what determines genetic diversity levels within species? PLoS Biol. 2012;10(9):e1001388.

35. Falk JE, Chan AC, Hoffmann E, Hochwagen A. A Mec1- and PP4-dependent checkpoint couples centromere pairing to meiotic recombination. Dev Cell. 2010;19(4):599-611.

Ready to submit your research? Choose BMC and benefit from:

- fast, convenient online submission

- thorough peer review by experienced researchers in your field

- rapid publication on acceptance

- support for research data, including large and complex data types

- gold Open Access which fosters wider collaboration and increased citations

- maximum visibility for your research: over $100 \mathrm{M}$ website views per year

At $\mathrm{BMC}$, research is always in progress.

Learn more biomedcentral.com/submissions 